\title{
Seletividade de herbicidas na cultura de crambe
}

\section{Selectivity of herbicides in crambe crop}

\author{
Guilherme Sasso Ferreira Souza ${ }^{1 *}$; Hermeson dos Santos Vitorino²; Ana Carolina \\ da Costa Lara Fioreze ${ }^{3}$; Maria Renata Rocha Pereira ${ }^{4}$; Dagoberto Martins ${ }^{5}$
}

\section{Resumo}

\begin{abstract}
A baixa produtividade do crambe pode estar associada a vários fatores e, dentre estes, a competição com as plantas daninhas, que reduz a produtividade da cultura, afeta a colheita e contribui para o aumento da umidade das sementes. Portanto, este estudo teve como objetivo avaliar a tolerância de plantas de crambe (cultivar FMS Brilhante) a diversos herbicidas graminicidas e latifolicidas aplicados em pré-plantio incorporado (PPI), em pré-emergência (PRÉ) e pós-emergência (PÓS). O estudo foi instalado em condições de casa-de-vegetação e os tratamentos testados constaram da aplicação: em PPI os herbicidas diclosulam, flumetsulam, metribuzim e trifluralin; em PRÉ os herbicidas atrazine, diclosulam, diuron, flumetsulam, metribuzim, S-metolachlor, sulfentrazone e trifluralin; e em PÓS os herbicidas bentazon, carfentrazone-ethyl, clefoxydim, cletodim+fenoxaprop-p-ethyl, ethoxysulfuron, fomesafen, fluazifop-p-butyl, flumioxazin, halosulfuron, imazamox, imazapic, lactofen, nicosulfuron, oxadiazon, quinclorac e setoxydim. Foram realizadas avaliações visuais de fitointoxicação nas plantas de crambe após as aplicações, as plântulas emergidas foram contabilizadas e ao final do período de avaliação a altura e a massa seca das plantas também foram determinadas. Nas condições em que os estudos foram conduzidos, pode-se concluir que apenas o herbicida trifluralin, aplicado em préemergência, e os herbicidas clefoxidim+fenoxaprop-p-ethyl, fluazifop-p-butyl, quinclorac, setoxydim e clefoxydim, aplicados em pós-emergência, apresentaram seletividade e potencial de uso para a cultivar de crambe FMS Brilhante.
\end{abstract}

Palavras-chave: Crambe abyssinica, modo de aplicação, fitotoxicidade, fitointoxicação

\begin{abstract}
The low productivity of crambe can be associated with many factors, among these, the competition with weeds, which reduces the yield, harvest affects and contributes to the increase in seed moisture. Therefore, this study aimed to evaluate the tolerance of crambe plants cv. FMS Brilhante to herbicides applied in preplant incorporated (PPI), preemergence (PRE), and postemergence (POST). The study was installed in a green-house and the treatments consisted of the herbicide application in: pre-plant incorporated ofdiclosulam, flumetsulam, metribuzin, and trifluralin;preemergence applicationof atrazine, diclosulam, diuron, flumetsulam, metribuzim, S-metolachlor, sulfentrazone, and trifluralin; and postemergence application ofbentazon, carfentrazone-ethyl, clefoxydim, cletodim + fenoxapropp-ethyl, ethoxysulfuron, fomesafen, fluazifop-p-butyl, flumioxazin, halosulfuron, imazamox, imazapic, lactofen, nicosulfuron, oxadiazon, quinclorac, and setoxydim. Visual evaluations of phytotoxicity on crambe plants were realized after applications, the seedlings were counted and the height and plant dry matter were determined in the end of the evaluation period. In conditions where the studies were conducted, we can conclude that only the trifluralin application in PRE and the application of

\footnotetext{
${ }^{1}$ Discente de Doutorado da Universidade Estadual Paulista “Júlio de Mesquita Filho”, UNESP, Botucatu, SP. E-mail: guisasso@ hotmail.com

2 Prof. Dr. da Universidade Estadual do Piauí, UESPI, Picos, PI. E-mail: vitorinohermeson@gamil.com

${ }^{3}$ Prof $^{\mathrm{a}} \mathrm{Dr}^{\mathrm{a}}$ da Universidade Federal de Santa Catarina, UFSC, Curitibanos, SC. E-mail: anacostlara@hotmail.com

${ }^{4}$ Pesquisadora Dr ${ }^{\mathrm{a}}$ da UNESP, Botucatu, SP. E-mail: mariarenata10@hotmail.com

${ }^{5}$ Prof. Dr. da UNESP, Botucatu, SP. E-mail: dmartins@fca.unesp.br

* Autor para correspondência
} 
clefoxidim+fenoxaprop-p-ethyl, fluazifop-p-butyl, quinclorac, setoxydim and clefoxydim in POST showed selectivity and potential use for FMS Brilhante crambe cultivar.

Key words: Crambe abyssinica, application mode, phytotoxicity, phytointoxication

\section{Introdução}

No cenário atual existe uma busca por fontes alternativas que venham suprir a demanda energética do mundo, pois as fontes como petróleo estão propensas a esgotarem-se. Nesse contexto, inúmeras espécies oleaginosas vêm sendo avaliadas para a produção dos biocombustíveis, com relação ao seu potencial em produção e qualidade do óleo. Uma dessas oleaginosas é o crambe (Crambe abyssinica Hochst), uma espécie da família Brassicaceae, sendo bem próximo a outras brássicas como canola e mostarda. A semente de crambe é a principal matéria prima da cultura, com cerca de $37 \%$ de óleo com uma composição de 55 a 60 \% de ácido erúcico (LESSMAN; BERRY, 1967). O óleo de crambe apresenta alta viscosidade e alto ponto de fumaça $o$ que o torna particularmente adequado para algumas utilizações industriais (LAZZERI et al., 1997), como a síntese de derivados químicos como a erucamida (na adição de alguns tipos de filmes plásticos), ácido brasílico (para a fabricação de nylon e polímeros) e derivados sulfurados (utilizados como aditivos para borrachas) (HINMAN, 1986).

O cultivo do crambe é uma opção interessante para a rotação de cultura com a soja, o milho, o trigo e outros grãos cultivados na região Sudeste, Sul e Centro-Oeste do Brasil. Apresenta ciclo curto, relativa tolerância à seca (GLASER, 1996) e baixo custo (PITOL, 2008). A introdução do crambe no Brasil teve início na decada de 90 por pesquisadores da Fundação MS - Maracajú, que após anos de pesquisa obtiveram a primera e única cultivar registrada no Brasil: 'FMS Brilhante'. Em condições brasileiras, a cultivar FMS Brilhante apresenta produtividade de grãos de 1000 a $1500 \mathrm{~kg}$ ha-1 (PITOL, 2008; FUNDAÇÃO MS, 2010; PITOL; BROCH; ROSCOE, 2010), o que é considerado abaixo do potencial produtivo da espécie Crambe abyssinica Hochst.
A baixa produtividade do crambe pode estar associada a vários fatores e, dentre estes, a ausência do controle das plantas daninhas, que reduz a produtividade da cultura, afeta a colheita e contribui para o aumento da umidade das semente (OPLINGER et al., 2008). Devido ao seu desenvolvimento inicial lento, durante as três ou quatro primeiras semanas após a emergência das plântulas, a competição com as plantas invasoras é crítica e a semeadura em áreas relativamente livres das mesmas é recomendado (ENDRES e SCHATZ, 2013). Assim, o objetivo do presente estudo foi avaliar a seletividade de vários herbicidas aplicados em pré-plantio incorporado, em pré-emergência e em pós-emergência sobre plantas de crambe.

\section{Material e Métodos}

O estudo foi instalado no município de Botucatu/ SP no período de Março a Agosto de 2011 e composto por dois experimentos, conduzidos em vasos de polietileno, com capacidade de 1,5 L, preenchidos com solo de barranco (Tabela 1) devidamente corrigido e adubado, em condições de casa-devegetação. Um experimento constou da aplicação de herbicidas em pré-plantio incorporado (PPI) e em pré-emergência (PRÉ) e o outro experimento da aplicação de herbicidas em pós-emergência (PÓS). Os tratamentos químicos utilizados nos dois estudos estão apresentados na Tabela 2.

As sementes de crambe utilizadas foram da cultivar FMS Brilhante, sendo estas semeadas a 1,5 $\mathrm{cm}$ de profundidade anteriormente a aplicação dos tratamentos em PRÉ e, para os tratamentos com aplicação em PPI as sementes foram semeadas a 1,5 cm de profundidade após a aplicação e incorporação dos herbicidas. A incorporação do herbicida com o solo nos tratamentos com aplicação em PPI foi realizada após a aplicação dos tratamentos através da mistura solo+herbicida em sacos de polietileno. 
Os tratamentos em PÓS foram aplicados quando as plantas apresentavam entre 4 e 6 folhas definitivas. Os dois experimentos foram instalados em um delineamento experimental inteiramente casualizado com quatro repetições, sendo também conduzidos vasos sem a aplicação de herbicidas para os três momentos de aplicação.

Tabela 1. Resultados da análise físico-química da amostra de solo utilizado no experimento. Botucatu/SP, 2011.

\begin{tabular}{|c|c|c|c|c|c|c|c|c|c|c|}
\hline \multirow{2}{*}{ SOLO } & \multirow{2}{*}{$\begin{array}{c}\mathrm{pH} \\
\mathrm{CaCl}_{2}\end{array}$} & \multirow{2}{*}{$\begin{array}{c}\text { M.O } \\
\mathrm{g} \mathrm{dm}^{-3}\end{array}$} & \multirow{2}{*}{$\begin{array}{l}\text { P resina } \\
\text { mg dm }^{-3}\end{array}$} & \multicolumn{6}{|c|}{$\mathrm{mmol} / \mathrm{dm}^{3}$} & \multirow{2}{*}{$\begin{array}{c}V \\
(\%)\end{array}$} \\
\hline & & & & $\mathbf{H}^{+}+\mathbf{A l}^{+3}$ & $\mathbf{K}^{+}$ & $\mathbf{C a}^{+2}$ & $\mathbf{M g}^{+2}$ & SB & CTC & \\
\hline LVd & 4,6 & 7 & 3 & 22 & 0,2 & 2 & 2 & 4 & 26 & 15 \\
\hline & $\frac{A}{65} \frac{1}{65}, 6$ & & $-\overline{\text { Silte }}$ & nulor & & 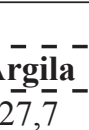 & & & $\bar{M}_{c}$ & \\
\hline
\end{tabular}

Fonte: Elaboração dos autores.

Tabela 2. Tratamentos químicos aplicados em pré-plantio incorporado, pré-emergência e pós-emergência sob plantas de crambe. Botucatu/SP, 2011.

\begin{tabular}{lcc}
\hline \multicolumn{1}{c}{ Tratamentos } & Dose (g $\left.\mathbf{i . a} \cdot \mathbf{~ h a}^{-1}\right)$ & Modo de Aplicação \\
\hline testemunha & --- & --- \\
atrazine & 1,500 & PRÉ $^{2}$ \\
diclosulam & 0,025 & PRÉ \\
diuron & 1,200 & PRÉ \\
flumetsulam & 0,105 & PRÉ \\
metribuzim & 0,360 & PRÉ \\
S-metolachlor & 1,200 & PRÉ \\
sulfentrazone & 0,500 & PRÉ \\
trifluralin & 1,140 & PRÉ \\
diclosulam & 0,025 & PPI \\
flumetsulam & 0,105 & PPI \\
metribuzim & 0,360 & PPI \\
trifluralin & 0,720 & PPI \\
bentazon & 0,720 & PÓS \\
carfentrazone-ethyl & 0,050 & PÓS \\
clefoxydim & 0,150 & PÓS \\
cletodim + fenox. ${ }^{3}$ & $0,040+0,040$ & PÓS \\
ethoxysulfuron & 0,080 & PÓS \\
fluazifop-p-butyl & 0,150 & PÓS \\
flumioxazin & 0,040 & PÓS \\
fomesafen & 0,225 & PÓS \\
halosulfuron & 0,112 & PÓS \\
imazamox & 0,242 & PÓS \\
imazapic & 0,098 & PÓS \\
lactofen & 0,250 & PÓS \\
nicosulfuron & 0,050 & PÓS \\
oxadiazon & 1,00 & PÓS \\
quinclorac & 0,375 & PÓS \\
setoxydim & 0,184 & PÓS \\
\hline
\end{tabular}

${ }^{1}$ cletodim + fenox. $=$ cletodim + fenoxaprop-p-ethyl. ${ }^{2}$ PRÉ-Pré-emergência. ${ }^{3} \mathrm{PPI}-$ Pré-plantio incorporado. ${ }^{4} \mathrm{PÓS}-\mathrm{Pó}$-emergência. Fonte: Elaboração dos autores. 
Para a aplicação dos herbicidas foi utilizado um pulverizador costal, pressurizado a $\mathrm{CO}_{2}$, equipado com uma barra de pulverização munida com quatro pontas de jato plano tipo XR 110.02VS, com pressão de trabalho de $200 \mathrm{kPa}$ que proporcionar um consumo de calda de $200 \mathrm{~L} \mathrm{ha}^{-1}$.

Para os tratamentos com aplicação em PPI e PRÉ foram realizadas avaliações visuais de fitointoxicação nas plantas de crambe aos 7, 14, 21, 28 e 35 dias após a aplicação (DAA) por meio uma escala percentual de notas, na qual 0 (zero) corresponde a nenhuma injúria demonstrada e 100 (cem) à morte das plantas, proposta pela Sociedade Brasileira da Ciência das Plantas Daninhas (1995). Foram contabilizadas as plântulas emergidas aos 7 , 14 e 21 DAA e, ao final do período de avaliação a altura a massa seca das plantas foram determinadas. Já, para os tratamentos com aplicação em PÓS foram realizadas avaliações visuais de fitointoxicação nas plantas de crambe aos 7, 14, 21 e 28 dias após a aplicação (DAA) através de uma escala percentual de notas, na qual 0 (zero) corresponde a nenhuma injúria demonstrada e 100 (cem) à morte das plantas, proposta pela SBCPD (1995) e, ao final do período de avaliação, 28 DAA, a massa seca das plantas foi determinada após secagem em uma estufa de ventilação forçada de ar a $65^{\circ} \mathrm{C}$ por $72 \mathrm{~h}$ com posterior pesagem.

Todos os resultados obtidos nos experimentos foram submetidos à análise de variância pelo Teste "F" e as médias dos tratamentos comparadas pelo teste de Tukey a $5 \%$ de probabilidade e os resultados do número de plantas emergidas foram transformados segundo a equação "y $=$ arco seno $\sqrt{ }$ $(\mathrm{x} / 100+0,5) "$.

\section{Resultados e Discussão}

Os resultados obtidos nas avaliações visuais de fitointoxicação para os tratamentos com aplicação em PRÉ e PPI estão apresentados na Tabela 3. Observase que, independentemente do ingrediente ativo ou da forma de aplicação dos herbicidas as plantas de crambe apresentaram sintomas visuais de injúria aos 7 DAA. O uso de sulfentrazone foi o tratamento que mais causou danos às plantas de crambe, proporcionando $89,50 \%$ de danos, já os demais tratamentos não proporcionaram danos superiores a $25 \%$. Aos 14 DAA verifica-se que os sintomas de injúrias apresentados pelas plantas de crambe foram incrementados em todos os tratamentos estudados, com exceção ao tratamento com aplicação de trifluralin em PRÉ, que proporcionou apenas 2,5\% de intoxicação as plantas.

$\mathrm{Na}$ avaliação realizada as 21 DAA nota-se maior elevação das porcentagens de fitointoxicação apresentadas pelas plantas de crambe submetidas a todos os tratamentos avaliados, atingindo valores mínimos de $55,50 \%$ de fitointoxicação, exceção feita aos tratamentos com aplicação de trifluralin em PRÉ e em PPI. No tratamento com aplicação de trifluralin em PRÉ as plantas de crambe recuperaram-se dos danos ocasionados nas avaliações anteriores e passaram a não apresentar mais sintomas de intoxicação, já no tratamento com aplicação de trifluralin em PPI os sintomas de injúrias aumentaram, porém ainda de forma branda em comparação aos demais herbicidas testados.

Verifica-se aos 28 DAA, como nas avaliações anteriores, que todos os herbicidas com exceção da aplicação de trifluralin em PRÉ continuaram a proporcionar injúrias crescentes as plantas de crambe, inclusive o tratamento com aplicação de trifluralin em PPI, no qual as plantas de crambe apresentaram sintomas visíveis de danos de $25 \%$. As plantas de crambe submetidas à aplicação de diuron, metribuzim e sulfentrazone em PRÉ e metribuzin em PPI apresentam 100\% de dano, ou seja, foram totalmente controladas por estes herbicidas. Stougaard e Moomaw (1991) avaliaram a injúria proporcionada pela aplicação de diversos herbicidas aplicados em PRÉ e em PPI incluindo trifluralin sobre plantas de crambe e, como ora observado neste estudo, também não notaram injúrias apenas nas plantas submetidas a aplicação de trifluralin em PRÉ. 
Tabela 3. Porcentagem de fitointoxicação de plantas de crambe submetidas à aplicação de herbicidas em préemergência e em pré-plantio incorporado. Botucatu/SP, 2011.

\begin{tabular}{|c|c|c|c|c|c|c|c|c|c|c|c|}
\hline \multirow{2}{*}{ Tratamentos } & \multirow{2}{*}{$\begin{array}{c}\text { Dose } \\
\left(\mathrm{g} \text { i.a. } \text { ha }^{-1}\right)\end{array}$} & \multicolumn{10}{|c|}{ Dias Após a Aplicação (DAA) } \\
\hline & & \multicolumn{2}{|c|}{7} & \multicolumn{2}{|c|}{14} & \multicolumn{2}{|l|}{21} & \multicolumn{2}{|c|}{28} & \multicolumn{2}{|l|}{35} \\
\hline 1.testemunha & --- & - & & -- & & --- & & - & & --- & \\
\hline 2. atrazine & 1,500 & 13,50 & $\mathrm{CD}$ & 39,75 & $\mathrm{DE}$ & 93,75 & A & 99,25 & A & 100,00 & A \\
\hline 3. diclosulam & 0,025 & 24,75 & B & 53,75 & $\mathrm{C}$ & 80,75 & $\mathrm{~B}$ & 92,00 & $\mathrm{AB}$ & 97,75 & A \\
\hline 4. diuron & 1,200 & 12,25 & $\mathrm{CD}$ & 48,00 & $\mathrm{CD}$ & 97,25 & $\mathrm{~A}$ & 100,00 & A & 100,00 & A \\
\hline 5. flumetsulam & 0,105 & 16,00 & $\mathrm{CD}$ & 45,25 & $\mathrm{CD}$ & 75,75 & $\mathrm{~B}$ & 88,25 & $\mathrm{~B}$ & 97,25 & A \\
\hline 6. metribuzim & 0,360 & 15,50 & $\mathrm{CD}$ & 32,50 & $\mathrm{EF}$ & 98,00 & $\mathrm{~A}$ & 100,00 & A & 100,00 & A \\
\hline 7. S-metolachlor & 1,200 & 17,75 & $\mathrm{BC}$ & 18,00 & $\mathrm{G}$ & 55,50 & $\mathrm{C}$ & 75,00 & $\mathrm{C}$ & 77,50 & $\mathrm{~B}$ \\
\hline 8. sulfentrazone & 0,500 & 89,50 & A & 98,75 & A & 99,50 & $\mathrm{~A}$ & 100,00 & A & 100,00 & A \\
\hline 9 trifluralin & 1,140 & 10,25 & D & 2,50 & $\mathrm{H}$ & 0,00 & $\mathrm{E}$ & 0,00 & $\mathrm{E}$ & 0,00 & $\mathrm{D}$ \\
\hline 10. diclosulam 1 & 0,025 & 23,50 & B & 73,00 & B & 76,50 & $\mathrm{~B}$ & 93,00 & $\mathrm{AB}$ & 96,75 & A \\
\hline 11. flumetsulam ${ }^{1}$ & 0,105 & 2,50 & E & 24,75 & $\mathrm{FG}$ & 78,50 & $\mathrm{~B}$ & 93,25 & $\mathrm{AB}$ & 96,50 & A \\
\hline 12. metribuzim ${ }^{1}$ & 0,360 & 19,50 & $\mathrm{BC}$ & 28,75 & $\mathrm{~F}$ & 99,75 & A & 100,00 & $\mathrm{~A}$ & 100,00 & A \\
\hline 13. trifluralin ${ }^{1}$ & 0,720 & 1,25 & E & 7,75 & $\mathrm{H}$ & 17,50 & $\mathrm{D}$ & 25,00 & $\mathrm{D}$ & 26,25 & $\mathrm{C}$ \\
\hline & & 238,75 & & 243,2 & & 680,22 & & 352,8 & & 552,92 & \\
\hline C.V. $(\%)$ & & 14,4 & & 8,9 & & 3,5 & & 4, & & 3,4 & \\
\hline d.m.s. & & 7,31 & & 8,62 & & 6,22 & & 8,6 & & 7,04 & \\
\hline
\end{tabular}

Médias seguidas da mesma letra não diferem estatisticamente entre si pelo teste $\mathrm{t}(\mathrm{p}<0,05)$.

${ }^{1}$ Pré-Plantio Incorporado.

** significativo a $1 \%$ de probabilidade.

Fonte: Elaboração dos autores.

$\mathrm{Na}$ última avaliação realizada, aos 35 DAA, registra-se que, com exceção do herbicida trifluralin aplicado em PRÉ as injúrias apresentadas pelas plantas de crambe submetidas a todos os demais tratamentos, tanto para os herbicidas aplicados em PRÉ quanto em PPI, foram elevadas e prejudiciais ao desenvolvimento das plantas de crambe.

$\mathrm{O}$ número de plantas emergidas, massa seca e altura das plantas de crambe submetidos à aplicação dos tratamentos em PRÉ e PPI estão apresentados na Tabela 4. Observa-se diferenças no número de plantas emergidas entre as contagens realizadas nos diferentes dias de avaliação, pois cada herbicida possui seu modo de ação e pode atuar em períodos diferentes, podendo assim ocorrer tratamentos com emergência semelhante a observada para a testemunha sem aplicação de herbicidas e estas plantas sofrerem os efeitos destes herbicidas tardiamente, como por exemplo para o diclosulam aplicado em PRÉ e em PPI.
Verifica-se que apenas o tratamento com aplicação de trifluralin em PRÉ mostrou-se semelhante à testemunha para as características altura de plantas e acúmulo de massa seca, reforçando assim a sua possibilidade de uso para esta cultura. Já, os demais tratamentos avaliados não apresentaram resultados satisfatórios para que possam ser utilizados na cultura do crambe, pois propiciaram reduções drásticas nas duas características avaliadas ou morte das plantas. Campbell e Walton (2007) observaram reduções de 43,64 e 10,91\% no crescimento de plantas de crambe submetidas a aplicação dos herbicidas diuron e S-metolachlor em pré-emergência, respectivamente. Observaram ainda, um acréscimo de $10,91 \%$ no crescimento de plantas de crambe quando submetidas a aplicação de trifluralin, o que não foi verificado quando da aplicação de trifluralin em pré-emergência. 
Tabela 4. Efeito de diferentes herbicidas aplicados em pré-emergência e em pré-plantio incorporado sobre o número de plântulas emergidas, altura e produção de massa de plantas de crambe. Botucatu/SP, 2011.

\begin{tabular}{|c|c|c|c|c|c|c|c|c|c|c|c|}
\hline \multirow{3}{*}{$\begin{array}{l}\text { Tratamentos } \\
\text { 1. testemunha }\end{array}$} & \multirow{3}{*}{$\begin{array}{c}\begin{array}{c}\text { Dose } \\
\left(\mathrm{kg} \text { i.a. } \text { ha-1 }^{-1}\right)\end{array} \\
---\end{array}$} & \multicolumn{6}{|c|}{ Número de plântulas emergidas ${ }^{2}$} & \multirow{2}{*}{\multicolumn{2}{|c|}{$\begin{array}{l}\text { Altura de } \\
\text { plantas } \\
(\mathrm{cm})\end{array}$}} & \multirow{2}{*}{\multicolumn{2}{|c|}{$\begin{array}{c}\text { Massa } \\
\text { seca } \\
(\mathrm{g})\end{array}$}} \\
\hline & & \multicolumn{2}{|c|}{ 7DAA $\mathbf{A}^{3}$} & \multicolumn{2}{|c|}{ 14DAA } & \multicolumn{2}{|c|}{ 21DAA } & & & & \\
\hline & & 14,75 & $\mathrm{C}$ & 56,75 & $\mathrm{AB}$ & 58,50 & $\mathrm{AB}$ & 6,15 & A & 1,61 & A \\
\hline 2. atrazine & 1,500 & 58,25 & $\mathrm{AB}$ & 71,50 & A & 14,75 & $\mathrm{CD}$ & 0,00 & $\mathrm{E}$ & 0,00 & $\mathrm{C}$ \\
\hline 3. diclosulam & 0,025 & 58,25 & $\mathrm{AB}$ & 63,25 & $\mathrm{AB}$ & 58,50 & $\mathrm{AB}$ & 0,65 & $\mathrm{DE}$ & 0,07 & $\mathrm{C}$ \\
\hline 4. diuron & 1,200 & 43,25 & $\mathrm{AB}$ & 61,50 & $\mathrm{AB}$ & 13,25 & $\mathrm{CD}$ & 0,00 & $\mathrm{E}$ & 0,00 & $\mathrm{C}$ \\
\hline 5. flumetsulam & 0,105 & 48,25 & $\mathrm{AB}$ & 59,75 & $\mathrm{AB}$ & 8,50 & $\mathrm{CD}$ & 1,14 & $\mathrm{CD}$ & 0,12 & $\mathrm{C}$ \\
\hline 6. metribuzim & 0,360 & 61,75 & A & 71,75 & $\mathrm{~A}$ & 41,50 & $\mathrm{BC}$ & 0,00 & $\mathrm{E}$ & 0,00 & $\mathrm{C}$ \\
\hline 7. S-metolachlor & 1,200 & 35,00 & $\mathrm{BC}$ & 48,25 & $\mathrm{~B}$ & 28,25 & $\mathrm{CD}$ & 1,65 & $\mathrm{C}$ & 0,20 & $\mathrm{C}$ \\
\hline 8. sulfentrazone & 0,500 & 13,25 & $\mathrm{C}$ & 20,00 & $\mathrm{C}$ & 5,00 & $\mathrm{D}$ & 0,00 & $\mathrm{E}$ & 0,00 & $\mathrm{C}$ \\
\hline 9. trifluralin & 1,140 & 41,50 & $\mathrm{AB}$ & 71,75 & A & 60,00 & $\mathrm{AB}$ & 6,90 & A & 1,61 & A \\
\hline 10. diclosulam ${ }^{1}$ & 0,025 & 43,25 & $\mathrm{AB}$ & 14,75 & $\mathrm{C}$ & 46,75 & $\mathrm{ABC}$ & 0,85 & $\mathrm{CDE}$ & 0,06 & $\mathrm{C}$ \\
\hline 11. flumetsulam ${ }^{1}$ & 0,105 & 38,25 & $\mathrm{ABC}$ & 16,75 & $\mathrm{C}$ & 56,50 & $\mathrm{AB}$ & 0,62 & $\mathrm{DE}$ & 0,08 & $\mathrm{C}$ \\
\hline 12. metribuzim ${ }^{1}$ & 0,360 & 48,25 & $\mathrm{AB}$ & 16,75 & $\mathrm{C}$ & 6,75 & $\mathrm{D}$ & 0,00 & $\mathrm{E}$ & 0,00 & $\mathrm{C}$ \\
\hline 13. trifluralin ${ }^{1}$ & 0,720 & 49,75 & $\mathrm{AB}$ & 11,75 & $\mathrm{C}$ & 66,50 & $\mathrm{~A}$ & 4,52 & $\mathrm{~B}$ & 0,91 & $\mathrm{~B}$ \\
\hline & & \multicolumn{2}{|c|}{$8.06^{* *}$} & \multicolumn{2}{|c|}{$36.18^{* *}$} & \multicolumn{2}{|c|}{$32.36^{* *}$} & \multicolumn{2}{|c|}{$164,17 * *$} & \multicolumn{2}{|c|}{$46,12 * *$} \\
\hline C.V. $(\%)$ & & \multicolumn{2}{|l|}{24.8} & \multicolumn{2}{|c|}{18.3} & \multicolumn{2}{|l|}{23.2} & \multicolumn{2}{|c|}{22,2} & \multicolumn{2}{|c|}{49,6} \\
\hline d.m.s. & & \multicolumn{2}{|l|}{26.28} & \multicolumn{2}{|l|}{20.51} & \multicolumn{2}{|l|}{20.68} & \multicolumn{2}{|l|}{0,96} & \multicolumn{2}{|l|}{0,45} \\
\hline
\end{tabular}

Médias seguidas da mesma letra não diferem estatisticamente entre si pelo teste $\mathrm{t}(\mathrm{p}<0,05)$.

${ }^{1}$ Pré Plantio Incorporado. ${ }^{2}$ Dados transformados segundo a equação "y = arco seno $\sqrt{ }(\mathrm{x} / 100+0,5)$ ". ${ }^{3} \mathrm{DAA}=$ Dias após a aplicação. ** significativo a $1 \%$ de probabilidade.

Fonte: Elaboração dos autores.

Verifica-se na Tabela 5 que todos os herbicidas avaliados, graminicidas ou latifolicidas, provocaram fitointoxicações às plantas de crambe, sendo os herbicidas latifolicidas os que mais provocaram injúrias visuais as plantas, com médias de danos acima de $89 \%$, exceção ao imazamox e ao ethoxysulfuron, que provocaram danos de 36,5 e $36,0 \%$, respectivamente. Nos tratamentos com aplicação de herbicidas graminicidas, com exceção ao tratamentos com aplicação de nicosulfuron, imazapic e halosulfuron, não se observa grandes danos as plantas, com no máximo $7,0 \%$ de fitointoxicação para as plantas tratadas com cletodim+fenoxaprrp-p-ethyl.

Aos 14 DAA nota-se uma elevação dos danos apresentados pelas plantas em todos os tratamentos avaliados, sendo que, alguns já passaram a proporcionaramorte das plantas, comooflumioxazin, bentazon, fomesafen, lactofen e carfentrazone-ethyl. O padrão de injúrias proporcionadas pelos herbicidas graminicidas manteve-se muito semelhante a avalicação anterior, com maiores porcentagens de controle para os tratamentos com nicosulfuron, imazapic e halosulfuron e menores porcentagens para os demais. Nas duas últimas avaliações visuais realizadas, aos 21 e 28 DAA, observa-se que os tratamentos que ja propporcionavam nas avaliações anteriores elavadas taxas de intoxicação as plantas, acabaram por controlá-las totalmente, fato este ocorrido com os herbicidas nicosulfuron, imazapic, halosulfuron, flumioxazin, bentazon, fomesafen, lactofen, oxadiazon, carfentrazoneethyl e ethoxysulfuron. $\mathrm{O}$ tratamento com aplicação do herbicida imazamox foi o único herbicida latifolicida que não controlou completamente as plantas de crambe, mas proporcionou intoxicação de $88,75 \%$. 
Tabela 5. Porcentagem de fitointoxicação e peso da massa seca $(\mathrm{g})$ apresentados por plantas de crambe pulverizadas com diferentes herbicidas em pós-emergência. Botucatu/SP, 2011.

\begin{tabular}{|c|c|c|c|c|c|c|}
\hline Tratamentos & $\begin{array}{c}\text { Dose } \\
\left(\mathrm{kg} \mathrm{i.a.} \text { ha-1 }^{-1}\right)\end{array}$ & $7 \mathbf{D A A}^{2}$ & 14 DAA & 21 DAA & 28 DAA & $\begin{array}{c}\text { Massa seca } \\
\text { (g) }\end{array}$ \\
\hline 1. testemunha & --- & --- & --- & --- & --- & $1,677 \quad \mathrm{AB}$ \\
\hline 2. bentazon & 0,720 & $97,75 \mathrm{~A}$ & $100,00 \mathrm{~A}$ & $100,00 \quad \mathrm{~A}$ & $100,00 \mathrm{~A}$ & $0,000 \mathrm{C}$ \\
\hline 3. carfentrazonel & 0,050 & $99,75 \quad \mathrm{~A}$ & $100,00 \mathrm{~A}$ & $100,00 \mathrm{~A}$ & $100,00 \mathrm{~A}$ & $0,000 \mathrm{C}$ \\
\hline 4. clefoxydim & 0,150 & $0,75 \mathrm{D}$ & $5,00 \mathrm{E}$ & $4,25 \mathrm{E}$ & $4,25 \mathrm{D}$ & $2,187 \quad \mathrm{~A}$ \\
\hline 5. cletodim + fenox. ${ }^{1}$ & $0,040+0,040$ & $7,00 \mathrm{D}$ & $10,00 \quad E$ & $6,75 \mathrm{E}$ & $5,50 \quad \mathrm{CD}$ & $1,231 \mathrm{ABC}$ \\
\hline 6. ethoxysulfuron & 0,080 & $36,00 \mathrm{C}$ & $96,25 \quad \mathrm{AB}$ & $98,75 \quad \mathrm{~A}$ & $100,00 \mathrm{~A}$ & $0,000 \quad \mathrm{C}$ \\
\hline 7. fluazifop-p-butyl & 0,150 & $4,00 \mathrm{D}$ & $9,75 \mathrm{E}$ & $6,00 \quad E$ & $4,50 \quad \mathrm{CD}$ & $2,247 \quad \mathrm{~A}$ \\
\hline 8. flumioxazin & 0,040 & $89,25 \quad \mathrm{~A}$ & $98,00 \quad \mathrm{AB}$ & $100,00 \mathrm{~A}$ & $100,00 \mathrm{~A}$ & $0,000 \quad \mathrm{C}$ \\
\hline 9. fomesafen & 0,225 & $99,75 \quad \mathrm{~A}$ & $100,00 \mathrm{~A}$ & $100,00 \mathrm{~A}$ & $100,00 \mathrm{~A}$ & $0,000 \mathrm{C}$ \\
\hline 10. halosulfuron & 0,112 & $52,50 \quad \mathrm{~B}$ & $89,75 \quad \mathrm{~B}$ & $96,25 \mathrm{AB}$ & $100,00 \mathrm{~A}$ & $0,000 \mathrm{C}$ \\
\hline 11. imazamox & 0,242 & $36,50 \quad \mathrm{C}$ & $55,75 \quad \mathrm{D}$ & $73,25 \quad \mathrm{D}$ & $88,75 \quad \mathrm{~B}$ & 0,509 BC \\
\hline 12. imazapic & 0,098 & $52,50 \quad \mathrm{~B}$ & $79,00 \quad \mathrm{C}$ & $90,50 \quad \mathrm{BC}$ & $100,00 \mathrm{~A}$ & $0,000 \quad \mathrm{C}$ \\
\hline 13. lactofen & 0,250 & $100,00 \mathrm{~A}$ & $100,00 \mathrm{~A}$ & $100,00 \quad \mathrm{~A}$ & $100,00 \mathrm{~A}$ & $0,000 \quad \mathrm{C}$ \\
\hline 14. nicosulfuron & 0,050 & $55,00 \quad \mathrm{~B}$ & $78,75 \mathrm{C}$ & $88,25 \mathrm{C}$ & $100,00 \mathrm{~A}$ & $0,000 \mathrm{C}$ \\
\hline 15. oxadiazon & 1,00 & $93,00 \mathrm{~A}$ & $99,00 \quad \mathrm{AB}$ & $100,00 \quad \mathrm{~A}$ & $100,00 \mathrm{~A}$ & $0,000 \quad \mathrm{C}$ \\
\hline 16. quinclorac & 0,375 & $2,25 \mathrm{D}$ & $7,50 \mathrm{E}$ & $10,00 \quad E$ & $12,25 \mathrm{C}$ & $2,249 \quad \mathrm{~A}$ \\
\hline 17. setoxydim & $\underline{0,184}$ & $1,50 \_$D & $3,25 \_E$ & $3,50 \_$E & 3,75_D & 1,926 A \\
\hline $\mathrm{F}_{\text {TRATAMENTO }}$ & & $186,032 * *$ & $478,461 * *$ & $1071,775^{*}$ & $850,935^{*}$ & $15,479 * *$ \\
\hline d.m.s. & & 11,5 & 9,73 & 6,74 & 7,81 & 1,26 \\
\hline C.V. (\%) & & 15,14 & 5,9 & 3,9 & 4,4 & 45,6 \\
\hline
\end{tabular}

Médias seguidas da mesma letra maiúscula na coluna e minúscula na linha não diferem estatisticamente entre si pelo teste de Tukey $(\mathrm{p}<0,05)$.

${ }^{1}$ cletodim + fenox. $=$ cletodim + fenoxaprop-p-ethyl. ${ }^{2}$ Dias após a aplicação. ${ }^{* *}$ significativo a $1 \%$ de probabilidade.

Fonte: Elaboração dos autores.

Os demais tratamentos apresentaram reduzidas porcentagens de intoxicação as plantas, sendo que as plantas tratadas com cletodim+fenoxaprop-pethyl, fluazifop-p-butyl e clefoxydim apresentaram redução dos sintomas de intoxicação nestas avaliações e, as plantas tratadas com os herbicidas quinclorac e setoxydim apresentaram apenas leve incremento nos danos aporesentados. Estes resultados evidenciam que esta cultura é muito sensível a aplicação de herbicidas, tanto latifolicidas quanto até mesmo graminicidas. Oliveira Neto et al. (2011) estudando a seletividade de plantas de crambe a herbicidas aplicados em pré-emergência verificaram que apenas o herbicida trifluralin em doses inferiores a $450 \mathrm{~g}$ i.a. ha $^{-1}$ demostrouse seletivo para a cultura. Stougaard e Moomaw (1991) avaliaram as injúrias da aplicação sequencial de imazethapyr $\left(0,033 \mathrm{~kg}\right.$ i.a. ha $\left.{ }^{-1}\right)+$ bentazon $\left(0,560 \mathrm{~kg}\right.$ i.a. ha- $\left.{ }^{-1}\right)+$ sethoydym $(0,224 \mathrm{~kg}$ i.a. ha$\left.{ }^{1}\right)$ e observaram de 55 a $83 \%$ de danos nas plantas submetidas a este tratamento.

Com relação ao acúmulo de massa seca das plantas ao final do estudo, registrou-se que onde houve morte das plantas de crambe a massa foi zero e, dentre os demais tratamentos apenas o com aplicação de imazamox não apresentou valores semelhantes a testemunha, demonstrando assim, que mesmo com algum dano causado pela aplicação dos herbicidas as plantas conseguiram desenvolverse normalmente durante o período de avaliação dos tratamentos.

Portanto, nas condições em que os estudos foram conduzidos, pode-se concluir que apenas o herbicida trifluralin, aplicado em pré-emergência, 
e os herbicidas clefoxidim+fenoxaprop-pethyl, fluazifop-p-butyl, quinclorac, setoxydim e clefoxydim, aplicados em pós-emergência, apresentaram seletividade e potencial de uso para a cultivar de crambe FMS Brilhante.

\section{Referências}

CAMPBELL, M. C.; WALTON, G. Alternative oilseed crops $x$ herbicide tolerance. Meckering: Newsletters, 2007. 221 p. Disponível em: <http://www.agric.wa.gov. au/objtwr/imported_assets/content/sust/biofuel/clima dafwa_alternativeoilseedsxherbicides_trial1.pdf $>$. Acesso em: 22 mar. 2012.

ENDRES, G.; SCHATZ, B. Crambe production. NDSU: Fargo, 2013. Disponível em: <http://www.ag. ndsu.edu/ pubs/plantsci/crops/a1010.pdf>. Acesso em: 22 jun. 2013.

FUNDAÇÃO MS. Tecnologias e produção: crambe. Maracaju: Fundação MS, 2010. 60 p.

GLASER, L. K. Crambe: an economic assessment of the feasibility of providing multiple-peril crop insurance. Washington: Economic Research Service for the Risk Management Agency, Federal Crop Insurance Corporation, 1996. 45 p.

HINMAN, C. M. Potential new crops. Scientific American, New York, v. 255, n. 1, p. 33-37, 1986.

LAZZERI, L.; MATTEI, F.; BUCELLI, F.; PALMIERI, S. Crambe oil: a potential new hydraulic oil and quenchant. Industrial Lubrication and Tribology, Amsterdam, v. 49, n. 2, p. 71-77, 1997.
LESSMAN, K. J.; BERRY, C. Crambe and vernonia research results at the forage farm in 1966. Lafayette: Purdue University, 1967. 3 p. (Agricultural Experiment Station, v. 284).

OLIVEIRA NETO, A. M.; GUERRA, N.; MACIEL, C. D. G.; SILVA, T. R. B.; LIMA, G. G. R. Seletividade de herbicidas aplicados em pré-emergência na cultura do crambe. Revista Brasileira de Herbicidas, Maringá, v. 10, n. 1, p. 49-56, 2011.

OPLINGER, E. S.; OELKE, E. A.; KAMINSKI, A. R.; PUTNAM, D. H.; TEYNOR, T. M.; DOLL, J. D.; KELLING, K. A.; DURGAN, B. R.; NOETZEL, D. M. Crambe. St. Paul: Alternative Field Crops Manual, 2008. Disponível em: <http://www.hort.purdue.edu/newcrop/ afcm/crambe.html>. Acesso em: 19 nov. 2011.

PITOL, C. Cultura do crambe. In: ROSCOE, R.; LOURENÇÃO, A. L. F.; GRIGOLLI, J. F. J.; MELOTTO, A. M.; PITOL, C.; MIRANDA, R. A. S. Tecnologia e produção: milho safrinha e culturas de inverno. Maracaju: Fundação MS, 2008. p. 85-88.

PITOL, C.; BROCH, D. L.; ROSCOE, R. Tecnologia e produção: crambe. Maracaju: Fundação MS, 2010. 60 p.

SOCIEDADE BRASILEIRA DA CIÊNCIA DAS PLANTAS DANINHAS - SBCPD. Procedimentos para instalação, avaliação e análise de experimentos com herbicidas. Londrina: SBCPD, 1995. 42 p.

STOUGAARD, R. N.; MOOMAW, R. S. Crambe (Crambe abyssinica) tolerance to herbicides. Weed Technology, Champaign, v. 5, n. 3, p. 566-569, 1991. 